\title{
SYNTHESIS OF NOVEL 4-(4-METHOXYBENZENE SULPHONYL)-3, 5-DIMETHYL/3-METHYL-5-PHENYL/3, 5- DIPHENYL/3, 5-DIMETHYL-1-PHENYL/3- METHYL -1, 5- DIPHENYL /1, 3, 5-TRIPHENYL PYRAZOLE
}

\author{
Sandhya Chhakra and S. S. Chauhan* \\ Chemistry Research Lab ABB GF 07, Department of Chemistry, School of Sciences, \\ Mody University of Science and Technology, Lakshmangarh-332311, Sikar, (Raj.), India \\ E-mail : sureshsingh2288@gmail.com
}

\begin{abstract}
An efficient synthesis of novel 3, 4, 5-trisubstituted pyrazole (2a-c) and 1, 3, 4, 5-tetrasubstituted pyrazole (2d-f) by the reaction of (1a-c) with $\mathrm{NH}_{2}-\mathrm{NH}_{2} /$ phenyl hydrazine in absolute $\mathrm{C}_{2} \mathrm{H}_{5} \mathrm{OH}$. The structure of the various pyrazole derivatives (2a-f) has been elucidated from elemental analysis and spectral studies viz. IR, NMR.

Keywords: $\beta$-diketone, hydrazine hydrate, phenyl hydrazine, sulphonyl group, and spectral studies.

(C) RASĀYAN. All rights reserved
\end{abstract}

\section{INTRODUCTION}

The five-membered heterocycles containing two adjacent nitrogen heteroatoms represent a class of important compounds. The pyrazole ring has pulled in much consideration as it has turned out to be genuinely available and indicates diverse biological activity. ${ }^{1,2}$ The significance of pyrazole lies in the way that they can be viably utilized as antiinflammatory ${ }^{3}$, anticonvulsant ${ }^{4}$, antibacterial agents ${ }^{5,6}$, antitumor ${ }^{7}$, antiviral ${ }^{8}$ and CNS depressant ${ }^{9}$.

The compound contains sulphonyl group which has been a focus of attention for their diversified biological activities ${ }^{10}$.Sulfones involve one of a kind position in the medication business with their bactericidal ${ }^{11,12}$ and antimalarial ${ }^{13}$ activity.

The marketed drugs of pyrazole, such as Ampyrone, Antipyrine, Dipyrone, Isofezolac, Lonazolac, and Pyrolan ${ }^{\circledR}$ have incredible therapeutic esteem, prompted the scan for fresher bioactive compounds of this class with 4-methoxybenzene sulphonyl, as one of the substituents in the ring.

\section{EXPERIMENTAL}

Melting points are uncorrected (open capillary tubes method). The IR spectra were recorded on a NicoletMegna FT-IR 550 spectrophotometer in $\mathrm{KBr}$ pellets. The ${ }^{1} \mathrm{H}-\mathrm{NMR}$ spectra were run on a BRUKER AVANCE II 400 NMR Spectrometer at 400.13 in $\mathrm{CDCl}_{3}$ using TMS as an internal standard. Impurity checked by thin layer chromatography. Satisfactory elemental analyses were obtained.

\section{Generalized Preparation}

Preparation of Substituted Pyrazole(2a-c) $)^{14-18}$

A mixture of 1a-c $\left(\beta\right.$-diketone ${ }^{19-20}, 2.70$ g., $\left.0.01 \mathrm{M}\right)$ and $\mathrm{NH}_{2}-\mathrm{NH}_{2}(0.5 \mathrm{~g} ., 0.01 \mathrm{M})$ was placed in a flask and refluxed in absolute $\mathrm{C}_{2} \mathrm{H}_{5} \mathrm{OH}(8 \mathrm{ml})$ for about eight hours on a warming mantle. The resultant reaction mixture was cooled and filtered. The crude product so obtained was recrystallized from $\mathrm{C}_{2} \mathrm{H}_{5} \mathrm{OH}$. Impurity in the compound was checked by thin layer chromatography using chloroform: methanol (9: 1), as the mobile phase.

Rasayan J. Chem., 12(1), 290-293(2019)

http://dx.doi.org/10.31788/RJC.2019.1215030

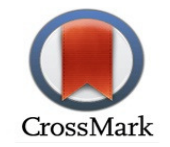


RASĀYAN J. Chem.

Vol. 12 | No. 1 |290 - 293| January - March | 2019

\section{Preparation of Substituted Pyrazole(2d-f)}

In a round-bottomed flask placed a mixture of $1 \mathrm{a}-\mathrm{c}(\beta$-diketone, $2.70 \mathrm{~g}$., $0.01 \mathrm{M})$ and phenyl hydrazine $(1.08 \mathrm{~g} .0 .01 \mathrm{M})$ and refluxed it in absolute ethanol $(10 \mathrm{ml})$ for approximately eight hours on a warming mantle. The resultant mixture was cooled and filtered. The crude product was recrystallized from ethanol. Impurity of the novel pyrazole derivative was confirmed through thin layer chromatography using ethyl acetate: $n$-hexane (8:2) as the mobile phase.

\section{RESULTS AND DISCUSSION}

Pyrazole derivatives (2a-f) were synthesized by the cyclocondensation of 1a-c with $\mathrm{NH}_{2}-\mathrm{NH}_{2} / \mathrm{NH}_{2} \mathrm{NH}-\mathrm{Ph}$ in the presence of absolute ethanol (Scheme-1). The pyrazole (2a-f) obtained were purified by column chromatography.
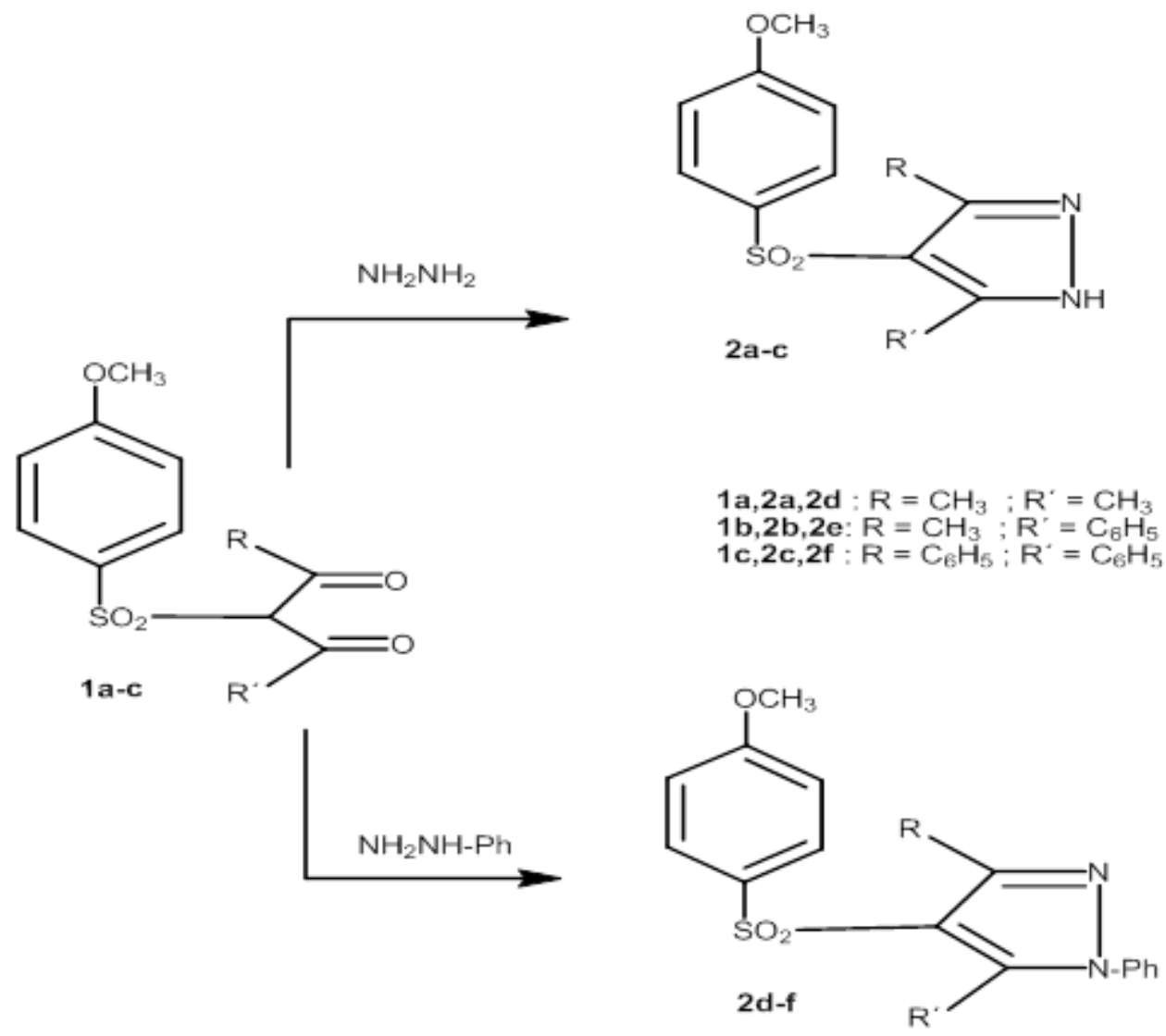

Scheme-1

Hydrazine hydrate/phenyl hydrazine other than being reactant make the media essential, to propel the enol kind of determined propane-1,3-dione(1a-c).The structure of all the recently combine pyrazolesubsidiaries (2a-f) was affirmed by the basic investigation, IR and ${ }^{1} \mathrm{H}$ NMR spectral studies(Table-1, 2 and 3).

\section{Spectral Studies}

In IR spectra, stretching vibration for $\mathrm{NH}$ observed at $3165-3260 \mathrm{~cm}^{-1}$ confirming $\mathrm{NH}$ group in the compound $(2 \mathrm{a}-\mathrm{c})$. The $\mathrm{C}=\mathrm{N}$ stretching mode appeared as an intense band at $1590-1620 \mathrm{~cm}^{-1}$ in fivemembered heterocyclic rings. A band at 1138-1150 and 1305-1340 $\mathrm{cm}^{-1}$ were attributed to symmetric and asymmetric $-\mathrm{SO}_{2}$ stretching vibration respectively. Absorption band also appeared at 1035-1060 and $1235-1265 \mathrm{~cm}^{-1}$, which was attributed to symmetric and asymmetric $-\mathrm{OCH}_{3}$ stretching vibrations (Table1).

In the ${ }^{1} \mathrm{H}$ NMR spectrum, a sharp singlet at $\delta 3.30-3.84$ accounted for three proton of $-\mathrm{OCH}_{3}$ group (2af). The methyl group at position-3 and 5 of the pyrazole ring was affirmed by a singlet at $\delta 2.04-2.05$ for 
RASĀYAN J. Chem.

Vol. 12 | No. 1 |290 - 293| January - March | 2019

six protons (2a \&2d). Another singlet at $\delta 1.98-2.01$ accounted for three protons of the methyl group attached to position 3 of pyrazole ring ( $2 \mathrm{~b} \& 2 \mathrm{e})$.

Table-1: IR Spectral Data $\left(\mathrm{cm}^{-1}\right)$ of New Pyrazole Derivative (2a-f)

\begin{tabular}{|c|c|c|c|c|c|c|c|c|}
\hline Compound & Ar-H & $\mathrm{C}-\mathrm{H}$ & \multicolumn{2}{|c|}{$\mathrm{O}-\mathrm{CH}_{3}$} & $\mathrm{C}=\mathrm{N}$ & $\mathrm{N}-\mathrm{H}$ & \multicolumn{2}{|c|}{$-\mathrm{SO}_{2}$} \\
\hline $2 \mathrm{a}$ & 3045 & 2910 & 1060 & 1250 & 1590 & 3245 & 1150 & 1300 \\
\hline $2 b$ & 3010 & 2940 & 1045 & 1240 & 1610 & 3165 & 1140 & 1340 \\
\hline $2 c$ & 3040 & - & 1050 & 1235 & 1580 & 3260 & 1150 & 1320 \\
\hline $2 \mathrm{~d}$ & 3035 & 2890 & 1058 & 1248 & 1620 & - & 1140 & 1310 \\
\hline $2 \mathrm{e}$ & 3050 & 2915 & 1035 & 1265 & 1600 & - & 1145 & 1305 \\
\hline $2 f$ & 3055 & - & 1040 & 1255 & 1620 & - & 1138 & 1308 \\
\hline
\end{tabular}

A signal at $\delta$ 7.21-7.33 showed the presence of one proton of $-\mathrm{NH}$ group in the pyrazole ring. A double doublet in the region $\delta 6.90-7.98$ accounted for the four aromatic protons (2a). A complicated pattern was observed at $\delta$ 6.88-8.04, which accounted for three phenyl groups (2f) attached to the 1, 3 and 5-position of the pyrazole ring and also other aromatic protons (Table-2). Satisfactory elemental analyses were obtained for all the compounds (Table-3).

Table-2: ${ }^{1} \mathrm{H}$ NMR Data $(\delta \mathrm{ppm})$ of Pyrazole Derivatives (2a-f)

\begin{tabular}{c|c|c|c|c}
\hline Compound & $\mathrm{Ar}-\mathrm{H}$ & $\mathrm{O}^{-\mathrm{CH}_{3}}$ & $\mathbf{H}_{3} \mathbf{C}_{\mathbf{C}}$ & $-\mathrm{NH}$ \\
& & & 2.05 & 7.30 \\
\hline $2 \mathrm{a}$ & $6.90-7.98$ & 3.84 & $(6 \mathrm{H}, \mathrm{s})$ & $(1 \mathrm{H}, \mathrm{b})$ \\
\hline $2 \mathrm{~b}$ & $(4 \mathrm{H}, \mathrm{dd})$ & $(3 \mathrm{H}, \mathrm{s})$ & 2.01 & 7.21 \\
& $6.93-7.83$ & 3.61 & $(3 \mathrm{H}, \mathrm{s})$ & $(1 \mathrm{H}, \mathrm{b})$ \\
\hline $2 \mathrm{c}$ & $(9 \mathrm{H}, \mathrm{m})$ & $(3 \mathrm{H}, \mathrm{s})$ & - & 7.33 \\
& $6.85-7.86$ & 3.71 & 2.04 & - \\
\hline $2 \mathrm{~d}$ & $(14 \mathrm{H}, \mathrm{m})$ & $(3 \mathrm{H}, \mathrm{s})$ & $(6 \mathrm{H}, \mathrm{s})$ & - \\
\hline $2 \mathrm{e}$ & $6.91-7.98$ & $(3 \mathrm{H}, \mathrm{s})$ & 1.98 & - \\
\hline $2 \mathrm{f}$ & $6.85-8.05(14 \mathrm{H}, \mathrm{m})$ & 3.81 & $(3 \mathrm{H}, \mathrm{s})$ & \\
\hline & & 3.30 & - & \\
\hline
\end{tabular}

Table-3: Physical Data of Pyrazole Derivatives (2a-f)

\begin{tabular}{|c|c|c|c|c|c|c|}
\hline \multirow[t]{2}{*}{ Compound } & \multirow[t]{2}{*}{ Molecular Formula } & \multirow{2}{*}{$\begin{array}{l}\text { M.P. } \\
\left({ }^{\circ} \mathrm{C}\right)\end{array}$} & \multirow{2}{*}{$\begin{array}{l}\text { Yield } \\
(\%)\end{array}$} & \multicolumn{3}{|c|}{ Elemental analysis Calculated (Found) \% } \\
\hline & & & & $\mathrm{C}$ & $\mathrm{H}$ & $\mathrm{N}$ \\
\hline \multirow[t]{2}{*}{$2 \mathrm{a}$} & $\mathrm{C}_{12} \mathrm{H}_{14} \mathrm{~N}_{2} \mathrm{O}_{3} \mathrm{~S}$ & 143 & 41 & 54.13 & 5.26 & 10.52 \\
\hline & & & & 53.68 & 4.92 & 10.06 \\
\hline \multirow[t]{2}{*}{$2 \mathrm{~b}$} & $\mathrm{C}_{17} \mathrm{H}_{16} \mathrm{~N}_{2} \mathrm{O}_{3} \mathrm{~S}$ & 182 & 39 & 62.19 & 4.87 & 8.53 \\
\hline & & & & 61.81 & 4.33 & 8.13 \\
\hline \multirow[t]{2}{*}{$2 \mathrm{c}$} & $\mathrm{C}_{22} \mathrm{H}_{18} \mathrm{~N}_{2} \mathrm{O}_{3} \mathrm{~S}$ & 190 & 43 & 67.69 & 4.61 & 7.18 \\
\hline & & & & 67.11 & 4.19 & 6.83 \\
\hline \multirow[t]{2}{*}{$2 \mathrm{~d}$} & $\mathrm{C}_{18} \mathrm{H}_{18} \mathrm{~N}_{2} \mathrm{O}_{3} \mathrm{~S}$ & 168 & 38 & 63.15 & 5.26 & 8.18 \\
\hline & & & & 62.78 & 4.89 & 7.73 \\
\hline \multirow[t]{2}{*}{$2 \mathrm{e}$} & $\mathrm{C}_{23} \mathrm{H}_{20} \mathrm{~N}_{2} \mathrm{O}_{3} \mathrm{~S}$ & 188 & 46 & 68.31 & 4.95 & 6.93 \\
\hline & & & & 67.91 & 4.53 & 6.51 \\
\hline \multirow[t]{2}{*}{$2 \mathrm{f}$} & $\mathrm{C}_{28} \mathrm{H}_{22} \mathrm{~N}_{2} \mathrm{O}_{3} \mathrm{~S}$ & 185 & 42 & 72.10 & 4.72 & 6.00 \\
\hline & & & & 71.68 & 4.35 & 5.49 \\
\hline
\end{tabular}

\section{CONCLUSION}

Compound (2a-f), have been effectively synthesized and characterized. A higher yield of compound (2af) was accomplished when refluxed approximately eight hours on a warming mantle at $120^{\circ}-125^{\circ} \mathrm{C}$. Elemental analysis and spectral (IR and ${ }^{1} \mathrm{H}$ NMR) results were good agreement with predicted formulae. 
RASĀYAN J. Chem.

Vol. 12 | No. 1 |290 - 293| January - March | 2019

\section{ACKNOWLEDGMENT}

Authors are thankful to the Dean (SOS), Mody University of Science and Technology, Lakshmangarh for providing necessary facilities to carry out present research work. Authors are also thankful to SAIF, Punjab University Chandigarh for giving spectral data.

\section{REFERENCES}

1. A. Gursoy, S. Demirayak, G. Capan, K. Erol, K. Vural, Euro. Jour. Med. Chem., 35(3), 359 (2000), DOI: $10.1016 / \mathrm{S} 0223-5234(00) 00117-3$

2. A. Mohammed, S. A. Khan, S. Srabu, J. India Chem. Soc., 79, 280 (2002)

3. A. K. Tiwari, V. P. Singh, P. Yadav, G. Gupta, A. Singh, R. K. Goel, P. Shinde, C. G. Mohan, Bioorganic Chemistry, 56, 8 (2014), DOI:10.1016/j.bioorg.2014.05.004

4. P. O. Patil, S. B. Bari, Arabian Journal of Chemistry, 9(4), 588 (2016), DOI:10.1016/j.arabjc.2013.08.027

5. A. Mohammed, S. M. Hasan, A. Wadoo, Oriental Journal of Chemistry, 18(2), 351(2002)

6. H.B'Bhatt, S.Sharma, Arabian Journal of Chemistry, 10(2), 1590(2017), DOI: $10.1016 /$ j.arabjc.2013.05.029

7. G. M. Nitulescu, C. Draghici, O. T. Olaru, Int. J. Mol. Sci., 14(11), 21805 (2013), DOI:10.3390/ijms141121805

8. B. K. Bhattacharya, R. K. Robins, G. R. Revankar, J. Heterocyclic Chemistry, 27(3), 795 (1990), DOI:10.1002/jhet.5570270358

9. P. V. Taberner, J. W. Unwin, J. Pharmacy and Pharmacology, 39(8), 658(1987), DOI: 10.1111/j.2042-7158.1987.tb03449.x

10. I. Ahmed, Shagufta, International Jour.of Pharmacy and Pharmaceutical Science,7(3), 19 (2015)

11. M. Moise, V. Sunel, L. Profire, M. Popa, C. Lionte, Farmacia, 56(3), 283 (2008)

12. E. A. Mohammad, M. Y. Mohammad, E. A. El-Bordany, Molecules, 18, 832(2013), DOI: $10.3390 /$ molecules 18010832

13. P. E. Thompson, B. Olszewski, J. A. Waitz, Am. J. Tropical Medicine Hyg., 14(3), 343 (1965), DOI:10.4269/ajtmh.1965.14.343

14. V. V. Dabholkar, F. Y. Ansari, J. Serb. Chem. Soc., 74(11), 1219 (2009), DOI: $10.2298 / J S C 0911219 D$

15. Y. C. Joshi, P. Joshi, S. S. Chauhan, S. Nigam, Heterocyclic Communications, 10, 249 (2004).

16. R. Fekry, N. A. Ismail, S. Raslan, European Chemical Bulletin, 5(5), 157 (2016), DOI:10.17628/ecb.2016.5.157-162

17. R. Unny, P. Joshi, M. P. Dobhal, Y. C. Joshi, Heterocyclic Communications, 2, 171 (2003)

18. S. S. Chauhan, A. Sharma, S. Saingar, Y. C. Joshi, J. Indian Chem. Soc., 82(11), 1016 (2005)

19. J. Bhagwan, Y. C. Joshi, R. P. Tyagi, B. C. Joshi, H. N. Mangal, J. Institution of Chemistry, 55, 58(1983)

20. S. S. Chauhan, Y. C. Joshi, An Indian J. Organic Chemistry, 5(4), 424(2009)

[RJC-5030/2018] 